Alessandro Moiraghi, $\mathrm{MD}^{\neq *}$ Francesco Prada, $\mathbf{M D}^{\S \uparrow \| *}$

Alberto Delaidelli, MD***

Ramona Guatta ${ }^{\ddagger}$

Adrien May ${ }^{\ddagger}$

Andrea Bartoli, MD ${ }^{\ddagger}$

Marco Saini, MD

Alessandro Perin, MD, PhD ${ }^{\S}$

Thomas Wälchli, MD,

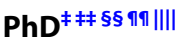

Shahan Momjian, MD ${ }^{\ddagger}$

Philippe Bijlenga, MD, PhD

Karl Schaller, MD ${ }^{\ddagger}$

Francesco DiMeco, MD ${ }^{\# \# ~}{ }^{* * * *}$

${ }^{\ddagger}$ Division of Neurosurgery, University of Geneva Faculty of Medicine, Geneva University Hospitals, Geneva, Switzerland; ${ }^{\S}$ Department of Neurosurgery, Fondazione IRCCS Istituto Neurologico "C. Besta," Milan, Italy; (Continued on next page)

*These authors contributed equally to this work.

Parts of the results have been presented as free communications at the Joint Annual Meeting of the Swiss Society of Neurosurgery and the Swiss Society of Neuroradiology on May 24, 2018, in Lugano, Switzerland.

\section{Correspondence:}

Francesco Prada, MD

Department of Neurosurgery,

Fondazione IRCCS Istituto Neurologico

"C. Besta", Milan, Italy.

Email: francesco.prada@istituto-besta.it

Received, November 1, 2018.

Accepted, April 16, 2019.

Copyright (C) 2019 by the

Congress of Neurological Surgeons

\title{
Navigated Intraoperative 2-Dimensional Ultrasound in High-Grade Glioma Surgery: Impact on Extent of Resection and Patient Outcome
}

BACKGROUND: Maximizing extent of resection (EOR) and reducing residual tumor volume (RTV) while preserving neurological functions is the main goal in the surgical treatment of gliomas. Navigated intraoperative ultrasound ( $\mathrm{N}$-ioUS) combining the advantages of ultrasound and conventional neuronavigation (NN) allows for overcoming the limitations of the latter.

OBJECTIVE: To evaluate the impact of real-time NN combining ioUS and preoperative magnetic resonance imaging (MRI) on maximizing EOR in glioma surgery compared to standard NN.

METHODS: We retrospectively reviewed a series of 60 cases operated on for supratentorial gliomas: 31 operated under the guidance of $\mathrm{N}$-ioUS and 29 resected with standard NN. Age, location of the tumor, pre- and postoperative Karnofsky Performance Status (KPS), EOR, RTV, and, if any, postoperative complications were evaluated.

RESULTS: The rate of gross total resection (GTR) in NN group was $44.8 \%$ vs $61.2 \%$ in $\mathrm{N}$-ioUS group. The rate of RTV $>1 \mathrm{~cm}^{3}$ for glioblastomas was significantly lower for the $\mathrm{N}$-ioUS group $(P<.01)$. In 13/31 (42\%), RTV was detected at the end of surgery with $\mathrm{N}$-ioUS. In 8 of 13 cases, ( $25.8 \%$ of the cohort) surgeons continued with the operation until complete resection. Specificity was greater in $\mathrm{N}$-ioUS ( $42 \%$ vs $31 \%$ ) and negative predictive value $(73 \%$ vs $54 \%$ ). At discharge, the difference between pre- and postoperative KPS was significantly higher for the $\mathrm{N}$-ioUS $(P<.01)$.

CONCLUSION: The use of an N-ioUS-based real-time has been beneficial for resection in noneloquent high-grade glioma in terms of both EOR and neurological outcome, compared to standard NN. N-ioUS has proven usefulness in detecting RTV $>1 \mathrm{~cm}^{3}$.

KEY WORDS: Extent of resection, Patient outcome, Gliomas, High-grade gliomas, Intraoperative ultrasound, Neuronavigation, Residual tumor volume

Operative Neurosurgery 0:1-11, 2019

DOI: 10.1093/ons/opz203

$\mathbf{T}$ he goal of the surgical treatment of cerebral high-grade gliomas (HGG) is maximizing the extent of resection (EOR) while preserving the functionality of relevant structures in order to ensure a good

ABBREVIATIONS: EOR, extent of resection; GBM, glioblastoma multiforme; GTR, gross total resection; HGG, high-grade glioma; iMRI, intraoperative MRl; KPS, Karnofsky Performance Status; LGG, low-grade glioma; MPrage, magnetization-prepared rapid gradient-echo; MRI, magnetic resonance imaging; $\mathrm{NN}$, neuronavigation; $\mathbf{N}$-ioUS, navigated intraoperative ultrasound; RTV, residual tumor volume; US, ultrasound; WHO, World Health Organization neurological outcome for the patient. $^{1-9}$ Increasing evidence suggests that maximizing the EOR and minimizing the residual tumor volume (RTV) are associated with improved patient outcome. ${ }^{3,6,7,10,11}$

In order to improve EOR and rates of complete resection, intraoperative image guidance plays a critical role, comprising several techniques such as neuronavigation (NN) imaging, intraoperative ultrasound (ioUS), or intraoperative magnetic resonance imaging (iMRI), as well as fluorescence techniques. ${ }^{2,8,12-23}$

ioUS shows some paramount advantages over other techniques, such as low cost, fast 
reproducibility during the same intervention to obtain realtime scans of the operative field, portability, and ease of use. In addition, ioUS offers the possibility to be combined with "advanced ultrasound techniques," such as contrast-enhanced ultrasound (US), navigated 3D US, and fusion imaging. ${ }^{24-31}$ ioUS has been reported to allow obtaining a gross total resection (GTR) in the majority of patients. ${ }^{32}$ Major pitfalls of ioUS technique include the lack spatial resolution, width and orientation of the field of view, and scan quality, all of which are strictly operator dependent. ${ }^{27}$ Thanks to fusion imaging, ioUS can be aligned with preoperative MRI for intraoperative $\mathrm{NN}$, allowing to overcome the individual limitations of simple US and classical NN. Navigated ioUS (N-ioUS) has been already validated for abdominal applications and recently successfully applied to neurosurgery. ${ }^{27,28,33,34}$ Despite these promising reports, only a few studies to date aimed at evaluating the impact of $\mathrm{N}$-ioUS on cerebral glioma EOR. ${ }^{35}$

The purpose of our work is understanding how $\mathrm{N}$-ioUS-guided surgery can impact EOR, rate of complete resection, and early neurological outcome at discharge in a series of patients with supratentorial HGG.

\section{METHODS}

\section{Patient Population}

We retrospectively evaluated the data of a series of 60 patients, who underwent supratentorial HGG surgical resection (Table 1). A total of 31 of those patients were operated under the assistance of a real-time $\mathrm{NN}$ system based on fusion imaging of intraoperative US and preoperative MRI (N-ioUS), at the Department of Neurosurgery, of Fondazione IRCCS Istituto Neurologico "C. Besta", Milan, Italy. A total of 29 patients were, instead, operated with the assistance of a standard NN, at the Department of Neurosurgery of Geneva University Hospitals, Geneva, Switzerland. On both sites, surgeries were performed, between October 2010 and December 2014, by board-certified neurosurgeons with at least $5 \mathrm{yr}$ of surgical experience. The institutional review board provided approval for this study, all patients were thoroughly informed about the surgical procedure, and written consent was obtained.

The 2 groups were compared in parallel. Pre- and postoperative Karnofsky Performance Status (KPS), pre- and postoperative tumor volume, EOR, and postoperative complications were evaluated.

\section{(Continued from previous page)}

"Department of Neurological Surgery, University of Virginia Health Science Center, Charlottesville, Virginia; "Focused Ultrasound Foundation, Charlottesville, Virginia; \#Department of Molecular Oncology, British Columbia Cancer Research Centre, Vancouver, Canada; ${ }^{* *}$ Department of Pathology and Laboratory Medicine, University of British Columbia, Vancouver, Canada; ${ }^{\neq \neq}$Group of CNS Angiogenesis and Neurovascular Link, Physician-Scientist Program, Institute for Regenerative Medicine, Neuroscience Center Zurich, University Hospital Zurich, Zurich, Switzerland; ${ }^{\S}$ Division of Neurosurgery, Department of Health Sciences and Technology, Swiss Federal Institute of Technology (ETH), University Hospital Zurich, Zurich, Switzerland; "ก Department of Fundamental Neurobiology, Krembil Research Institute, University of Toronto, Toronto, Canada; IIIIDivision of Neurosurgery, Department of Surgery, Toronto Western Hospital, Toronto, Canada; \#\# Department of Pathophysiology and Transplantation, University of Milan, Milan, Italy; *** Department of Neurological Surgery, Johns Hopkins Medical School, Baltimore, Maryland

\begin{tabular}{|c|c|}
\hline $\mathbf{N}$ & $\begin{array}{c}\text { Overall } \\
60\end{array}$ \\
\hline Age (mean [SD]) & $55.32(14.84)$ \\
\hline \multicolumn{2}{|l|}{ Gender } \\
\hline Male (\%) & $38(63.3)$ \\
\hline Female (\%) & $22(36.7)$ \\
\hline \multicolumn{2}{|l|}{ Gliomas } \\
\hline WHO III (\%) & $9(15.0)$ \\
\hline WHO grade IV (\%) & $51(85.0)$ \\
\hline N-ioUS (\%) & $31(51.7)$ \\
\hline NN (\%) & $29(48.3)$ \\
\hline \multicolumn{2}{|l|}{ Localization } \\
\hline Noneloquent (\%) & $40(66.7)$ \\
\hline Eloquent (\%) & $20(33.3)$ \\
\hline Tumor volume $\mathrm{cm}^{3}$ (mean [SD]) & $36.69(31.16)$ \\
\hline Preoperative KPS (median [IQR]) & $80.00[77.50,90.00]$ \\
\hline
\end{tabular}

The 2 groups were comparable considering the variables investigated (Table 2). All patients performed a preoperative T1 gadolinium magnetization-prepared rapid gradient-echo (MPrage) MRI sequence (Siemens, Munich, Germany) for surgical planning and postoperative MRI evaluation of EOR, as explained below. Patients were subgrouped according to tumor location: all patients with tumors in eloquent areas (primary motor cortex, primary visual cortex, and speech areas) were operated on with asleep neurophysiological monitoring techniques (motor evoked potentials, sensory evoked potentials, and cortical and subcortical stimulation), whereas others were operated under general anesthesia. Histological diagnosis was obtained to confirm the diagnosis of HGG. Eloquent and noneloquent lesions were separately evaluated (Table 1).

\section{N-ioUS Technique Assessment}

In the N-ioUS group, we used an US system (MyLab Twice, Esaote, Genova, Italy) equipped with Fusion Imaging for Virtual Navigator (MedCom GmbH, Darmstadt, Germany) to obtain the fusion of 2D US images with the coplanar preoperative MRI. ioUS imaging at Fondazione IRCCS Istituto Neurologico "C. Besta" was performed directly by surgeons (Drs Prada and DiMeco) with expertise and training in US. Preoperative images were acquired by 3 T MRI volumetric, T1weighted, gadolinium contrast-enhanced, and fluid attenuated inversion recovery sequences. The US is equipped with an electromagnetic virtual navigation system for instrument tracking. The probe used is a linear transducer with variable band with a bandwidth of 3 to $11 \mathrm{MHz}$ (Esaote LA332, Genova, Italy), protected with a sterile cover (CIVCO, Coralville, Iowa) for the implementation of ioUS scan. The technique of ioUS and preoperative MRI fusion was performed as a rigid registration of external bony landmarks with a pointer. The reference for electromagnetic navigation was then placed on the US probe in order to continue the navigation in pair with US images. This technique allows to plan a craniotomy based on preoperative MRI images as every NN tools. Once the bone flap is removed, a first US control is made, before opening the dura, to check the accuracy. This also allows for correction of the brain shift according to intracranial landmarks, in order to realign US and MRI (fine-tuning) and to restart the navigation process with coplanar images. 
TABLE 2. Comparison between NN and N-ioUS Groups

\begin{tabular}{|c|c|c|c|c|}
\hline $\mathbf{n}$ & $\begin{array}{c}\text { NN } \\
29\end{array}$ & $\begin{array}{c}\text { N-ioUS } \\
31\end{array}$ & $\mathbf{p}$ & Test \\
\hline Age (mean [SD]) & $56.31(15.41)$ & $54.39(14.48)$ & 0.62 & Student $\mathrm{t}$ \\
\hline \multicolumn{5}{|l|}{ Gender } \\
\hline Male (\%) & $19(65.5)$ & $19(61.3)$ & 0.793 & Exact \\
\hline Female (\%) & $10(34.5)$ & $12(38.7)$ & & (Fisher) \\
\hline \multicolumn{5}{|l|}{ Gliomas } \\
\hline WHO III (\%) & $2(7.0)$ & $7(22.6)$ & 0.2731 & Exact \\
\hline WHO IV (\%) & $27(93.0)$ & $24(77.4)$ & & (Fisher) \\
\hline \multicolumn{5}{|l|}{ Localization } \\
\hline Noneloquent (\%) & $20(69.0)$ & $20(64.5)$ & 0.788 & Exact \\
\hline Eloquent (\%) & $9(31.0)$ & $11(35.5)$ & & (Fisher) \\
\hline Tumor volume $\mathrm{cm}^{3}$ (mean [SD]) & $36.21(29.64)$ & $37.14(33.00)$ & 0.909 & Student $\mathrm{t}$ \\
\hline Preoperative KPS (median [IQR]) & $80.00[80.00,90.00]$ & $80.00[65.00,90.00]$ & 0.281 & Nonnorm (Wilcox) \\
\hline
\end{tabular}

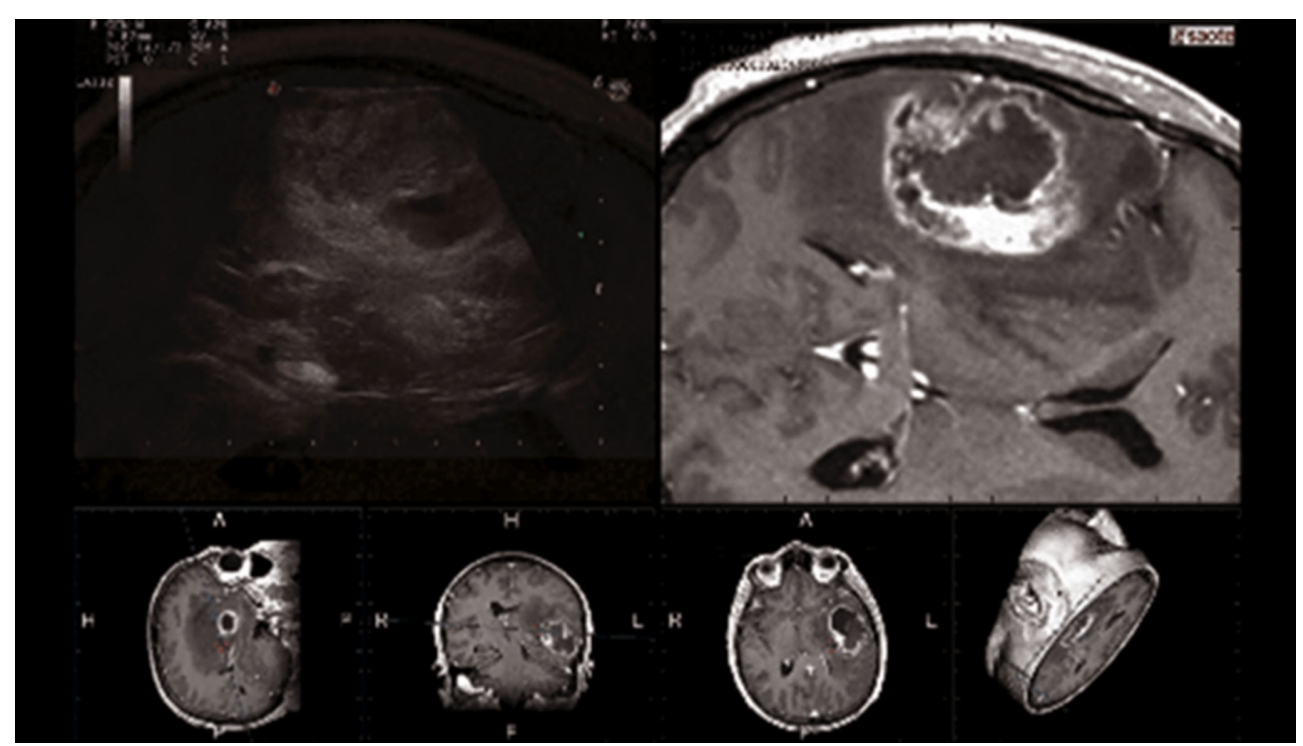

FIGURE 1. B-mode ioUS scan performed after the removal of the bone flap in a case of left temporal GBM. Ultrasound (left) fused in "overlapping mode" with a contrast-enhanced, T1-weighted preoperative MRI (right). In both images aside from the lesion, perilesional edema and adjacent cerebral structures, such as the lateral ventricles, thalamus, head of the caudate nucleus, and lenticular, nucleus can be seen.

A more extensive description of this technique is reported in published work. ${ }^{27,28,33}$

In all cases, a craniotomy was performed under the guidance of virtual navigation system. After bone flap removal, a first control was performed with $\mathrm{N}$-ioUS on closed dura, to correctly identify the lesion and surrounding structures (Figure 1). Any misalignment of the images was reported and corrected with fine-tuning operation (Figure 2), a tool allowing to freeze both US or MRI images and to realign them, to restart navigation with a restored accuracy. This maneuver can be repeated several times during surgery (every time that the surgeon detects a loss of accuracy), and a multiplanar correction can also be performed. Thanks to these operations, the surgeon is able to reduce brain-shift effects on navigation, as previously described. ${ }^{27,28}$ The identification of potential residual tumor, respective to the apparently complete resection, was reported by the operators in their records for every patient included in the study. In case the $\mathrm{N}$-ioUS detected residual tumor tissue on navigated B-mode images, this was considered as a true residual and surgery was completed or stopped according to the ioUS findings, depending on the eloquent or noneloquent tumor location and according to the surgeon's judgment.

\section{NN Control Group}

All patients in the NN group were operated on with the guidance of standard NN (Brainlab, Munich, Germany). All craniotomies were 


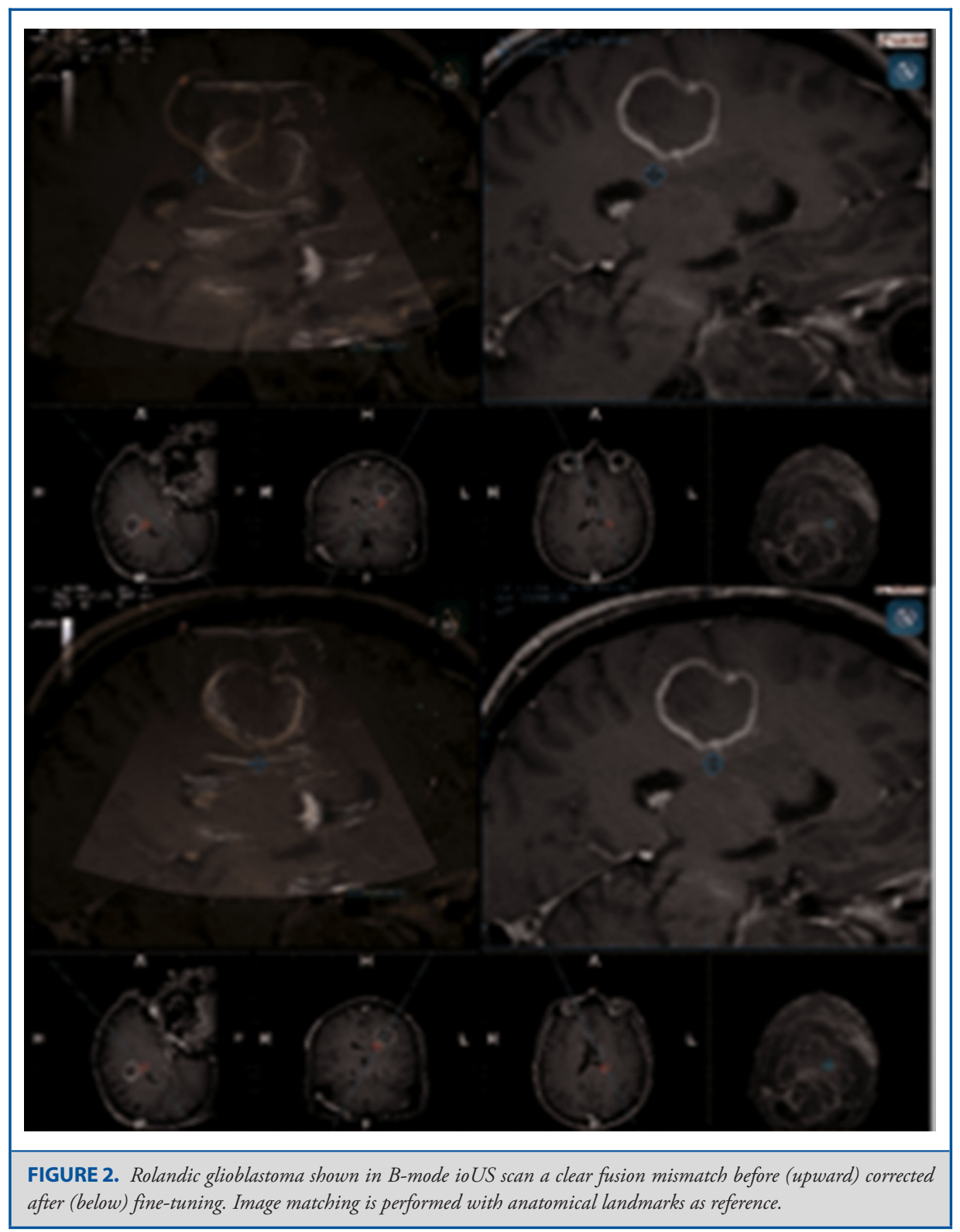

planned and tailored to the lesion according to NN. At the end of the intradural part of the intervention $\mathrm{NN}$, we used to perform a last control of resection margins and check their accordance to preoperative MRI.

\section{EOR and Outcome}

The EOR was calculated by comparing MRI studies performed before surgery with early postoperative MRI studies (within $48 \mathrm{~h}$ ). T1 MPrage contrast-enhancing volume was manually segmented on each section of MRI studies using a dedicated software (Iplannet, Brainlab, Munich, Germany). As previously described, the EOR was calculated using the following formula:

[(Preoperative Volume - Postoperative Volume) / Preoperative Volume] $\mathrm{x} 100 .^{11}$
GTR was considered as EOR $=100 \%$. Thus, we divided the patients into 3 groups according to the degree of resection: GTR, EOR $\geq 90 \%$, and EOR $<90 \%$. The assessment of tumor volumes was performed by A.M. (fourth year resident in neurosurgery) and validated by F.P. (board certified neurosurgeon). Data on patients' outcomes were collected from neurosurgeons' medical records at the time of presentation and discharge letters, with particular care for new onset neurological deficits and differences between post- and preoperative KPS $(\triangle \mathrm{KPS}){ }^{8,11}$

\section{Statistical Analysis}

Data are represented as mean + SEM, unless otherwise specified. The statistical analysis was performed with the Mann-Whitney $U$-test 


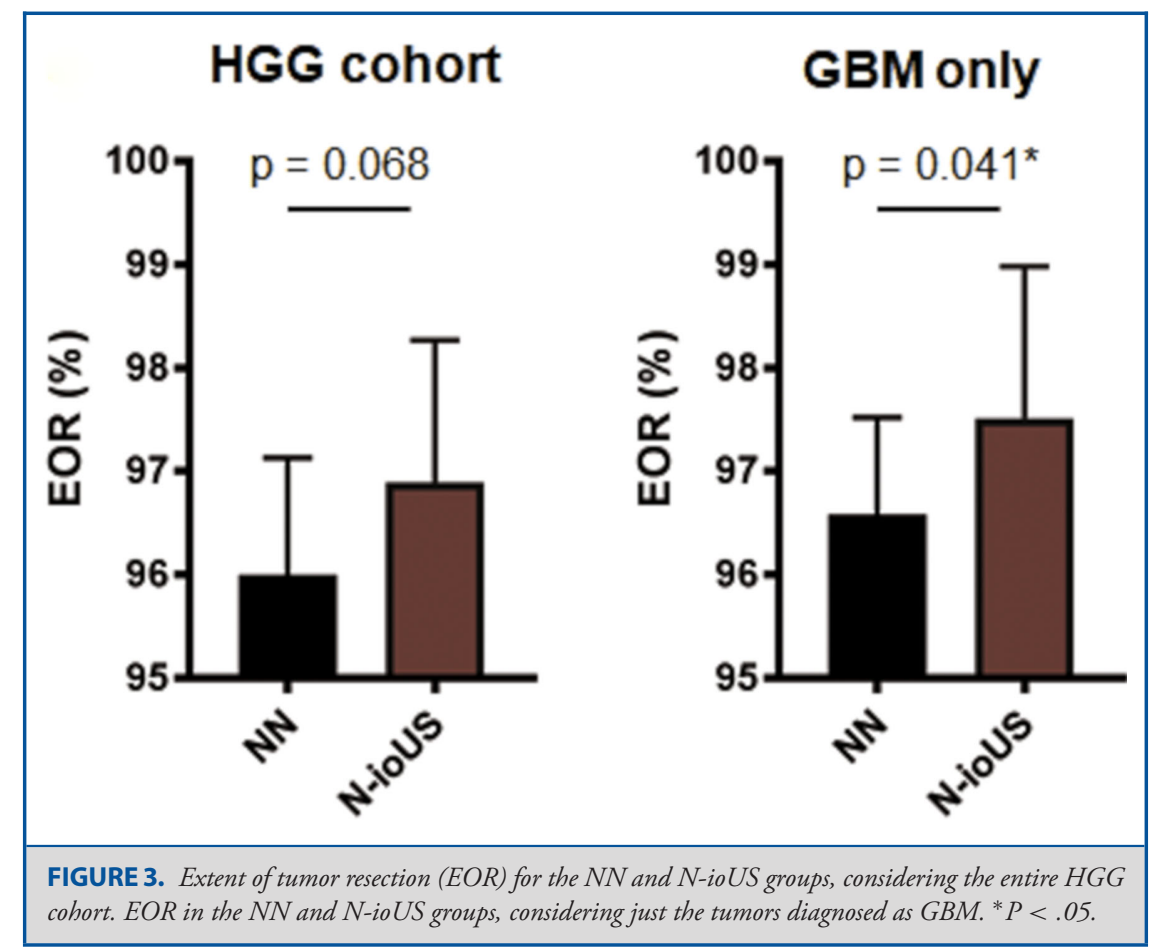

and Fisher's exact test. Statistical analysis was performed using GraphPad Prism 6 (GraphPad Software, San Diego, California).

\section{RESULTS}

A total of 60 patients were included ( 22 females and 38 males, with a mean age of $55 \mathrm{yr}$, range 18-77) with a mean preoperative KPS of 80 (median 80, range 40-100). The sample includes a total of 9 World Health Organization (WHO) grade III and 51 WHO grade IV (39 primary diagnosed glioblastoma multiforme [GBM] and 12 recurrent GBM). The detailed clinical characteristics of the cohort are summarized in (Table 1).

\section{Craniotomy Planning}

In all cases, the craniotomy was correctly positioned, showing no differences between the 2 groups. In all 31 cases studied with $\mathrm{N}$-ioUS, fine-tuning was performed immediately after the removal of bone and/or during resection, in order to correct the displacement between the images caused by brain shift. No complications in the operative field have been reported in both groups.

\section{EOR and RTV}

The 2 patient groups ( $\mathrm{NN}$ and $\mathrm{N}$-ioUS) were comparable for age, sex, tumor localization (eloquent or noneloquent), preoperative tumor volume, and preoperative clinical presentation (Table 2).
We obtained an EOR average of $96 \%$ in the NN group and $97 \%$ in the N-ioUS group with no statistical significance for HGG, but significance for GBM $(P<.04)$ (Figure 3$)$. The rate of GTR in the NN group was $44.8 \%$, and EOR $<90 \%$ was $10.3 \%$, whereas in the N-ioUS group a $61.2 \%$ GTR rate was obtained with a $6.4 \%$ rate of EOR $<90 \%$ (Figure 4). Considering only GBM, we found $44.4 \%$ rate of GTR in the NN group and $66.6 \%$ in the $\mathrm{N}$-ioUS group, and $11.1 \%$ of EOR $<90 \%$ in the NN group compared with $4.1 \%$ in the $\mathrm{N}$-ioUS group (Figure 4).

A postsurgical RTV is one of the most important predictors of poor patient outcome in GBM. ${ }^{7,11}$ In order investigate the role of $\mathrm{N}$-ioUS in minimizing the RTV, we compared this value in the 2 cohorts. The rate of RTV $>1 \mathrm{~cm}^{3}$ for GBMs was significantly lower for the $\mathrm{N}$-ioUS group $(P<.01$, Figure 5$)$ compared to the NN group. In order to better understand the impact of N-ioUS on GBM surgery, we performed the same analysis just for lesions affecting eloquent areas, operated under the guidance of functional mapping techniques with the assistance of imaging techniques like N-ioUS an NN. As a comparison, we also performed the same analysis for lesions in noneloquent areas, in which the resection was only guided by $\mathrm{N}$-ioUS or NN. This analysis revealed that the $\mathrm{N}$-ioUS group had a significant lower rate of postoperative RTV $>1 \mathrm{~cm}^{3}(P<.02)$, but this was not significant for eloquent lesions $(P<.1$, Figure 5$)$. Furthermore, we registered one case of noneloquent GBM in the NN group, for which the postoperative MRI showed a $2.85 \mathrm{~cm}^{3} \mathrm{RTV}$, so that the patient had to undergo a second surgery to achieve complete resection. No cases for which a second surgery was required were 

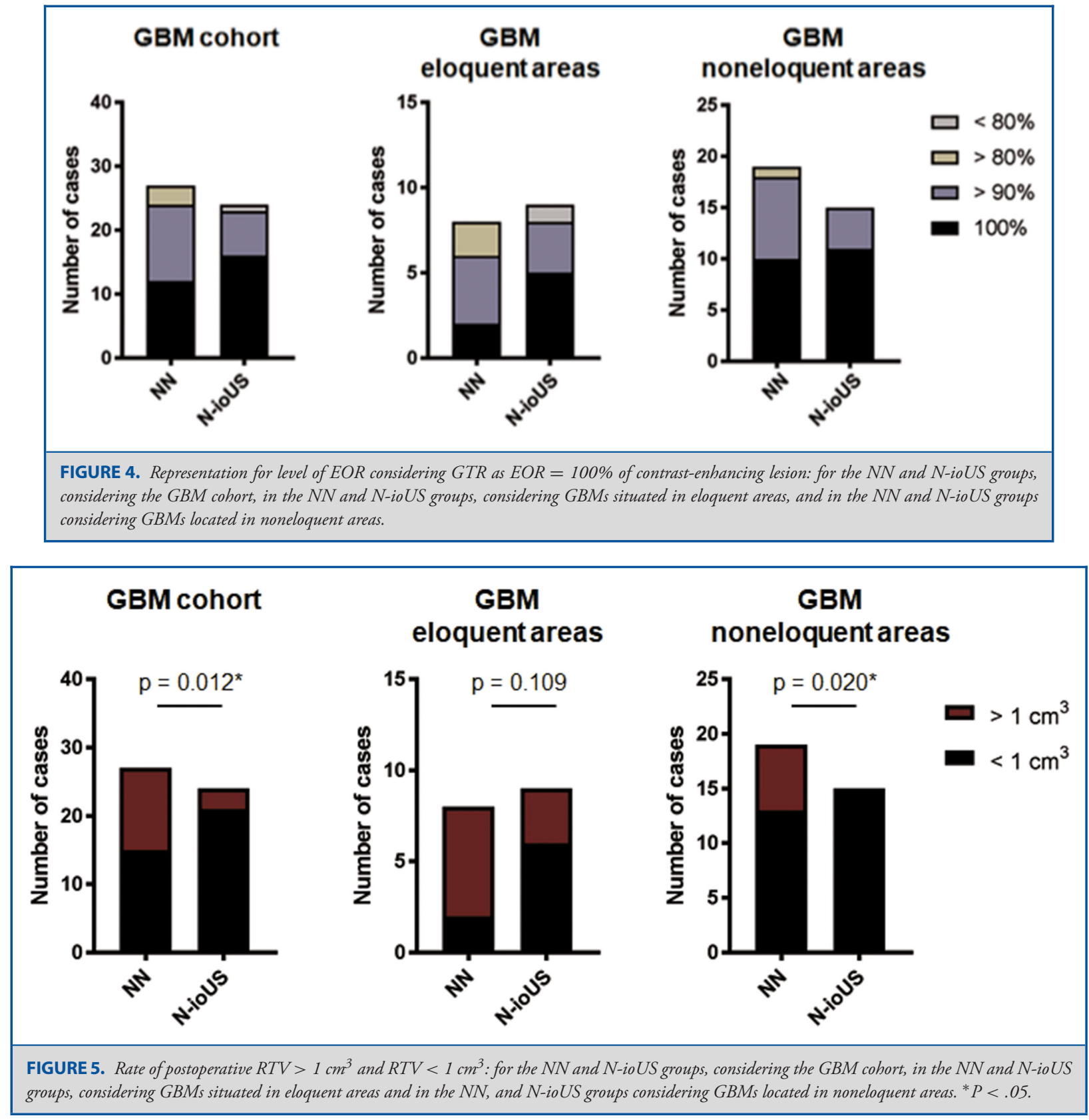

registered in the $\mathrm{N}$-ioUS group. Collectively, these data indicate that $\mathrm{N}$-ioUS, compared to $\mathrm{NN}$ alone, could be particularly useful in minimizing the RTV in noneloquent GBMs

\section{Complications and Outcome}

Intraoperative complications were observed in none of the 2 groups. For 5 cases $(16 \%)$ in the $\mathrm{N}$-ioUS group, we observed additional neurological deficits in the immediate postoperative period, and 3 patients showed transient minor deficits, with a rapid improvement of symptoms within a few postoperative days and a complete recovery at discharge. In 2 cases, postoperative complications occurred, but were likely not related to the use of US technique: one case of rigor nucalis and hyperpyrexia with negative blood and urine cultures, which resolved after treatment with intravenous vancomycin and ceftriaxone, and one case of hematemesis likely due to steroid therapy. In the $\mathrm{NN}$ group, we registered 7 cases $(24.1 \%)$ of postoperative neurological deficits. A 75-yr-old patient had no neurological changing after the intervention, but died of a massive pulmonary embolism. 


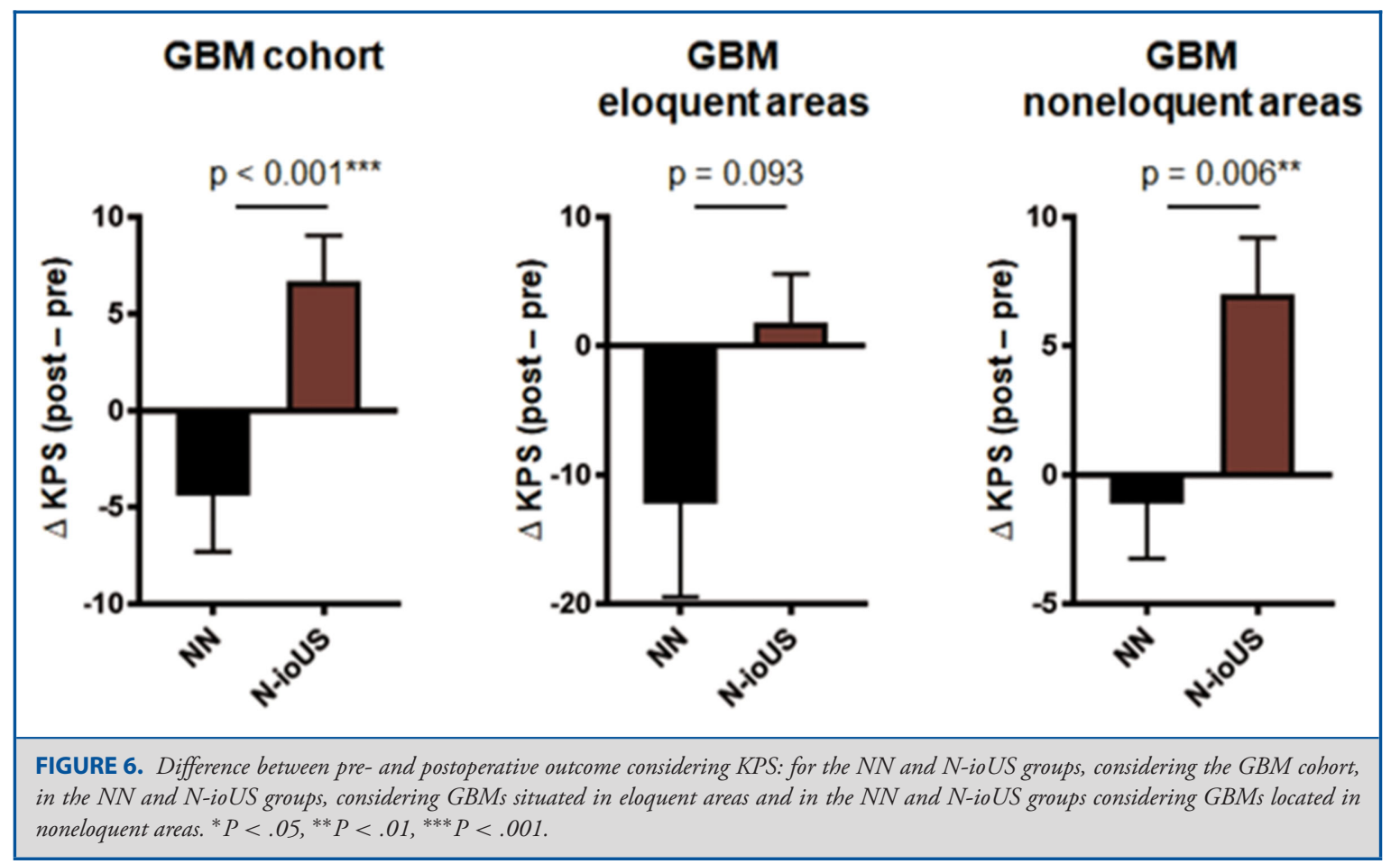

At discharge, the $\triangle$ KPS was significantly higher for the $\mathrm{N}$-ioUS $(P<.01)$, particularly in the noneloquent subgroup $(P<.01)$, a similar trend was observed in the eloquent subgroup, but not significant $(P<.1$; Figure 6$)$.

\section{$\mathbf{N}$-ioUS Findings}

In $13 / 31(42 \%)$, RTV was detected at the end of surgery with $\mathrm{N}$-ioUS. In 8 of 13 cases ( $25.8 \%$ of the cohort), surgeons continued with the operation until complete resection was documented with $\mathrm{N}$-ioUS. In the remaining 5 cases, the identified residual tumor could not be removed because of its position close to eloquent structures. In all of these 5 cases, the presence of residual tumor was confirmed by postoperative MRI, with a postoperative average volume of $9.63 \mathrm{~cm}^{3}$ (range 2.37$\left.14.74 \mathrm{~cm}^{3}\right)$.

In a group of 26 of 31 patients, apparent complete resection was assumed from the US control. In 19 of these 26 (73\%), the postoperative MRI confirmed complete removal of the tumor mass (Figures 7 and 8). In the remaining 7 of 26 cases (26.9\%) in which the US assessment was contradicted by the finding of residual tumor on postoperative MRI (false negatives), the mean residual volume was an average $0.46 \mathrm{~cm}^{3}\left(\right.$ range $\left.0.25-0.82 \mathrm{~cm}^{3}\right)$, obtaining a mean $\mathrm{EOR}=98.1 \%$, whereas in the $\mathrm{NN}$ subgroup, false-negative RTV was an average $1.82 \mathrm{~cm}^{3}$ (range 0.24-6.71 $\mathrm{cm}^{3}$ ) with a mean $\mathrm{EOR}=95 \%$.

In our series, we found a greater specificity for identifying RTV intraoperatively in the $\mathrm{N}$-ioUS group ( $42 \%$ vs $31 \%$ ). Negative predictive value for RTV of the N-ioUS was also higher compared to the $\mathrm{NN}$ group ( $73 \%$ vs $54 \%$ ).

\section{DISCUSSION}

An increasing number of studies have reported the usefulness of ultrasonography in identifying glial lesions and guide their resection, with one of the first reports being presented by Unsgaard et al, ${ }^{26,31,36-38}$ who reported 114 intracranial lesions operated under 2D ioUS guidance between 1997 and 2001.

\section{Resection Rates}

We obtained a postoperative GTR of $61.2 \%$ in the NioUS patients against the $44.8 \%$ of the NN group in a series of 60 nonselected consecutively operated supratentorial HGG. According to literature, several groups have reported higher EOR using $\mathrm{NN}$, opposed to surgery without NN. ${ }^{12,39}$ In a few studies, the assessment of the EOR for gliomas operated with ioUS has shown encouraging results, although it must be considered that these studies are often conducted using nonvolumetric measurements. ${ }^{40,41}$ Solheim et al ${ }^{42}$ have reported a $37 \%$ rate of GTR in a series of 156 malignant gliomas consecutively operated with $3 \mathrm{D}$ ioUS, with a worsening of neurological functions to 4 to $6 \mathrm{wk}$ in $13 \%$ of cases. In a subgroup of patients with unifocal lesions, contrast-enhancing, noninvasive basal ganglia, thalamus, or corpus callosum, and with KPS $>70$, the percentage of cases with GTR grows to $63 \%{ }^{42}$ In a study of Moiyadi et al ${ }^{43}$ the use of 3D ioUS in a series of 51 HGG has led to obtain a GTR in 


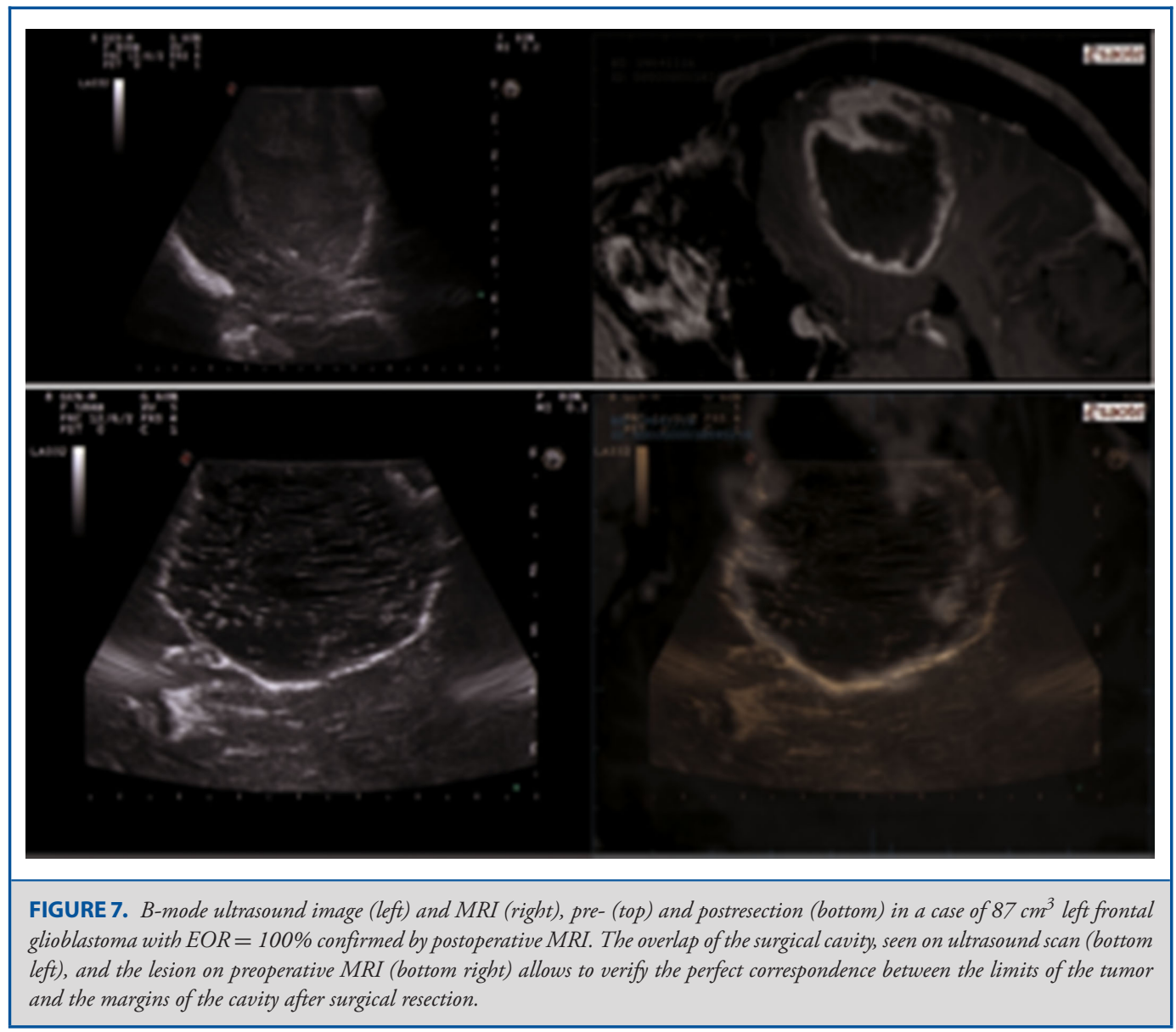

$47 \%$ of cases, with a frequency of complications similar to those obtained with other intraoperative imaging techniques. Dividing the series in resectable and unresectable tumors, the fraction of GTR increased up to $88 \%$ for resectable lesions, and was reduced to $24 \%$ for unresectable ones. ${ }^{43}$ In a recently published study, Šteňo et $\mathrm{al}^{44}$ reported an increased resection rate in low-grade glioma (LGG) resection combining 3D ioUS to awake resection techniques, compared to a previous series of patients operated in awake surgery and the only aid of standard NN. Despite the limitations of our study and of the published literature, having a real-time intraoperative reference during glioma surgery seems beneficial for both LGG and HGG.

\section{RTV and Outcome}

Not only the EOR but also increased residual volume appears to be an independent prognostic factor for reduced survival in HGG. ${ }^{1,6-8,11}$ US technique seems to be more accurate than standard NN for identifying residual tumor, with excellent sensitivity for volumes greater than $2 \mathrm{~cm}^{3}$, but with reduced accuracy for volumes smaller than $1 \mathrm{~cm}^{3}$.39,40 The better accuracy of iMRI in detecting small residues $<1 \mathrm{~cm}^{3}$ has already been reported in the literature. ${ }^{40}$ Consistently with these studies, we reported an advantage in favor of N-ioUS in detecting RTV $>1 \mathrm{~cm}^{3}$ in noneloquent GBM. In a recent prospective study, Coburger et $\mathrm{al}^{45}$ reported that modern linear high-frequency ioUS reaches a degree of accuracy close to iMRI in detecting residual tumor masses during intraoperative resection control in HGG. Sensitivity was even higher in linear array ioUS (76\%) than in iMRI (55\%) without significant differences in specificity for both. ${ }^{45}$ In our N-ioUS cohort, in 7 of 26 cases with assumed intraoperative complete resection, a residual tumor could be detected by postoperative MRI control (average volume of $0.46 \mathrm{~cm}^{3}$ ). Specificity and positive predictive value of $\mathrm{N}$-ioUS were higher than $\mathrm{NN}$ in our series. As ioUS helps differentiating between tumor and healthy brain tissue, ioUS can help achieving a maximal resection while providing safety.

In 13 of 31 cases, the $\mathrm{N}$-ioUS evaluation at the end of resection allowed to identify residual tumor, which could not be identified by NN. For 8 of these cases, the surgery continued and resection was completed according to $\mathrm{N}$-ioUS, reaching an MRI-confirmed GTR without additional neurological deficits for the patients. Only 1 out of these 8 patients presented a neurological deficit 

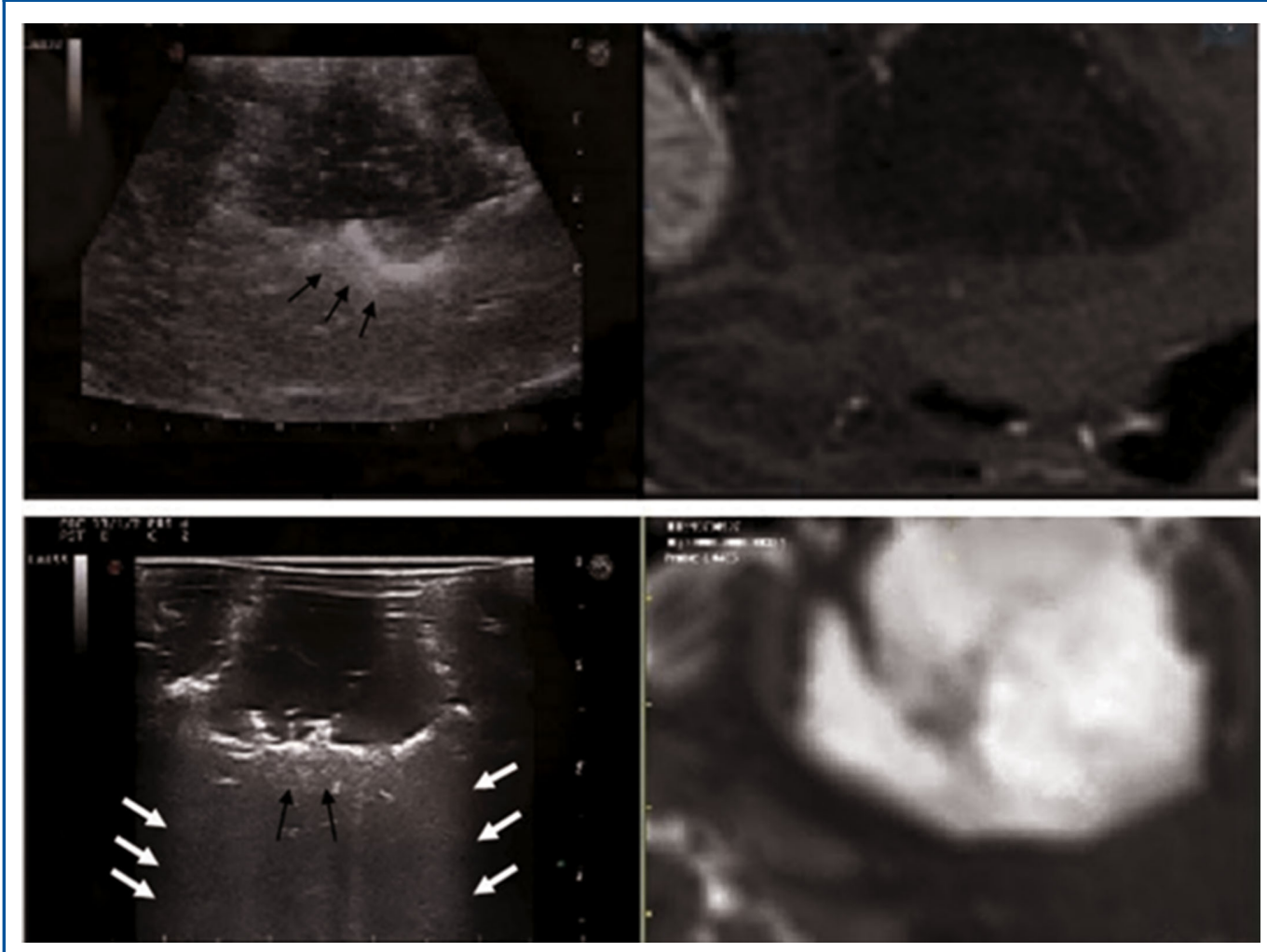

FIGURE 8. B-mode ultrasound scans (left images) and preoperative MRI images (right images) at the end of resection in a case of glioblastoma (above) and fibrillary astrocytoma (below). In both cases, a hyperechoic area on the bottom of the surgical cavity (black arrows) can be observed, associated with the presence of a residual tumor mass, and confirmed later by postoperative MRI. In the lower left image, the residue is partially hidden by the presence of back reinforcement artifacts behind the operative cavity (white arrows), due to the presence of blood and surgical manipulation. In this case, the comparison with the preoperative images (right) helps the operator in the interpretation of a doubtful image.

in the immediate postoperative (mixed aphasia), showing a rapid improvement until discharge. For the remaining 5 of these 13 cases, it was not possible to go beyond the actual resection, and the average residual volume was $9.63 \mathrm{~cm}^{3}$. N-ioUS has proven to be safe showing, in noneloquent localization, a significantly better postoperative $\triangle \mathrm{KPS}$, compared to the $\mathrm{NN}$ control group. The onset of neurological deficits in the immediate postoperative period in our series was similar to what reported in the literature. ${ }^{46}$

\section{Limitations}

The main limitation of this work resides in its retrospective nature. We attempted to limit the retrospective bias associated with this approach by confirming the EOR with pre- and postoperative MRI, reducing selection bias and not selecting the groups of patients based on age, tumor size and location, and preoperative conditions of patients. The use of a control group from a different institution is also a limitation of our study. However, it would be nearly impossible, outside of a prospective clinical trial, having 2 cohorts of patients from the same institution undergoing
2 different surgical treatments without considering different time periods. We privileged studying a series of patients operated during the same period with the same standards (even if it remains a limitation), instead of considering the cases operated by the same surgeons before starting their experience with ioUS. This last possibility would have probably augmented the experience bias, as well as introduced a considerable difference concerning patients' data quality.

\section{Technical Considerations}

$\mathrm{N}$-ioUS was employed for surgical planning and craniotomy placing, to identify the lesion before dural opening and during tumor resection, for brain-shift correction, and for the detection of any residual tumor at the end of surgery. For all $31 \mathrm{~N}$-ioUS group cases, it was necessary to use fine-tuning during the operation, after the removal of the operculum bone, and, subsequently, during intradural surgery in order to correct the discrepancies between ioUS and MRI images caused by brain shift. In all these cases relying on US images, the surgeon was 
always able to correct navigation misalignment. Recalibration and fusion of US imaging and MRI has demonstrated an excellent ability to compensate the loss of accuracy due to brain shift. ${ }^{28} \mathrm{On}$ the other hand, at the end of most of the surgeries, a substantial distortion and deformation of brain parenchyma often makes it impossible to have a precise overlap between images. In this case, the surgeon relies exclusively on US images. In cases of important parenchymal deformation, it would have been impossible to complete any intervention relying solely on images from the initial registration because of the increasing navigation loss of accuracy, possibly emphasizing the drawbacks of the classical NN.

The pursuit of a radical resection seems feasible thanks to fluorescence-guided surgery, but obtaining more complete and aggressive resections can augment the risks of a neurological deterioration of the patient. ${ }^{16,17,47,48}$ The authors believe that the use of an intraoperative imaging technique such as $\mathrm{N}$-ioUS coupled with advanced US imaging techniques such as contrastenhanced US and elastography ${ }^{49-51}$ will allow a well-trained neurosurgeon to overcome the limitations of standard B-mode imaging, fostering the understanding of different US techniques. Ultimately, the use of ioUS in synergy with intraoperative fluorescence techniques might lead to higher resection rates with reduced morbidity, and future studies are needed to clarify the exact potential of this combination.

\section{CONCLUSION}

Despite the limitations of the study, N-ioUS has proved beneficial for the resection of HGG located in noneloquent areas, in terms of both EOR and postoperative neurological outcome. In our experience, $\mathrm{N}$-ioUS appears to be reliable in identifying RTV with volumes $>1 \mathrm{~cm}^{3}$, whereas, for smaller volumes, the accuracy of US is decreased.

\section{Disclosures}

The authors have no personal financial or institutional interest in any of the drugs, materials, or devices described in this article.

\section{REFERENCES}

1. Hardesty DA, Sanai N. The value of glioma extent of resection in the modern neurosurgical era. Front Neur. 2012;18;3:140. (doi: 10.3389/fneur.2012.00140).

2. Hervey-Jumper S, Berger M. Role of surgical resection in low- and high-grade gliomas. Curr Treat Options Neurol. 2014;16(4):284.

3. Lacroix M, Abi-Said D, Fourney DR, et al. A multivariate analysis of 416 patients with glioblastoma multiforme: prognosis, extent of resection, and survival. J Neurosurg. 2001;95(2):190-198.

4. Sanai NN, Berger MSMS. Glioma extent of resection and its impact on patient outcome. Neurosurgery. 2008;62(4):753-766.

5. Sanai N, Berger M. Recent surgical management of gliomas. Adv Exp Med Biol. 2012;746:12-25. (doi: 10.1007/978-1-4614-3146-6_2).

6. Smith J, Chang E, Lamborn K, et al. Role of extent of resection in the longterm outcome of low-grade hemispheric gliomas. J Clin Oncol. 2008;26(8):13381345.

7. Brown TJ, Brennan MC, Li M, et al. Association of the extent of resection with survival in glioblastoma a systematic review and meta-analysis. JAMA Oncol. 2016;2(11):1460-1469.
8. Duffau H, Moritz-Gasser S, Gatignol P. Functional outcome after language mapping for insular World Health Organization Grade II gliomas in the dominant hemisphere: experience with 24 patients. Neurosurg Focus. 2009;27(2):E7.

9. Chambless LB, Kistka HM, Parker SL, Hassam-Malani L, McGirt MJ, Thompson RC. The relative value of postoperative versus preoperative Karnofsky Performance Scale scores as a predictor of survival after surgical resection of glioblastoma multiforme. J Neurooncol. 2015;121(2):359-364.

10. Sanai N, Polley M-Y, McDermott MW, Parsa AT, Berger MS. An extent of resection threshold for newly diagnosed glioblastomas. J Neurosurg. 2011;115(1): 3-8.

11. Fukui A, Muragaki Y, Saito T, et al. Volumetric analysis using low-field intraoperative magnetic resonance imaging for 168 newly diagnosed supratentorial glioblastomas: effects of extent of resection and residual tumor volume on survival and recurrence. World Neurosurg. 2017;98:73-80. (doi: 10.1016/j.wneu.2016.10.109).

12. Rainer Wirtz WS, Albert FK, Schwaderer M, et al. The benefit of neuronavigation for neurosurgery analyzed by its impact on glioblastoma surgery. Neurol Res. 2000;22(4):354-360.

13. Woydt M, Krone A, Becker G, Schmidt K, Roggendorf W, Roosen K. Correlation of intra-operative ultrasound with histopathologic findings after tumour resection in supratentorial gliomas. Acta Neurochir. 1996;138(12):1391-1398.

14. Stummer W, Pichlmeier U, Meinel T, Wiestler O, Zanella F, Reulen H. Fluorescence-guided surgery with 5-aminolevulinic acid for resection of malignant glioma: a randomised controlled multicentre phase III trial. Lancet Oncol. 2006;7(5):392-401.

15. Stummer W, Reulen HJ, Meinel T, et al. Extent of resection and survival in glioblastoma multiforme: identification of and adjustment for bias. Neurosurgery. 2008;62(3):564-576.

16. Höhne J, Schebesch K-M, de Laurentis C, et al. Fluorescein sodium in the surgical treatment of recurrent glioblastoma multiforme. World Neurosurg. 2019. (doi:10.1016/J.WNEU.2019.01.024).

17. Acerbi F, Broggi M, Schebesch KM, et al. Fluorescein-guided surgery for resection of high-grade gliomas: a multicentric prospective phase II study (FLUOGLIO). Clin Cancer Res. 2018. (doi:10.1158/1078-0432.CCR-17-1184).

18. Erdoan N, Tucer B, Mavl E, Menkü A, Kurtsoy A. Ultrasound guidance in intracranial tumor resection: correlation with postoperative magnetic resonance findings. Acta Radiol. 2005;46(7):743-749.

19. Kubben PL, ter Meulen KJ, Schijns OEMG, ter Laak-Poort MP, van Overbeeke JJ, van Santbrink H. Intraoperative MRI-guided resection of glioblastoma multiforme: a systematic review. Lancet Oncol. 2011;12(11):1062-1070.

20. Nimsky C, Fujita A, Ganslandt O, Von Keller B, Fahlbusch R. Volumetric assessment of glioma removal by intraoperative high-field magnetic resonance imaging. Neurosurgery. 2004;55(2):358-371.

21. Orringer D, Lau D, Khatri S, et al. Extent of resection in patients with glioblastoma: limiting factors, perception of resectability, and effect on survival. J Neurosurg. 2012;117(5):851-859.

22. Sanai N, Mirzadeh Z, Berger MS. Functional outcome after language mapping for glioma resection. $N$ Engl J Med. 2008;358(1):18-27.

23. Willems PWA, Taphoorn MJB, Burger H, van der Sprenkel JWB, Tulleken CAF. Effectiveness of neuronavigation in resecting solitary intracerebral contrastenhancing tumors: a randomized controlled trial. J Neurosurg. 2006;104(3):360368.

24. Sidhu PS, Cantisani V, Dietrich CF, et al. The EFSUMB guidelines and recommendations for the clinical practice of contrast-enhanced ultrasound (CEUS) in non-hepatic applications: update 2017 (short version). Ultraschall Med. 2018;39(2):154-180.

25. Prada F, Del Bene M, Saini M, Ferroli P, DiMeco F. Intraoperative cerebral angiosonography with ultrasound contrast agents: How I do it. Acta Neurochir. 2015;157(6):1025-1029.

26. Unsgaard G, Gronningsaeter A, Ommedal S, Hernes TAN. Brain operations guided by real-time two-result of improved image quality. Neurosurgery. 2002;51(2):402-412.

27. Prada F, Del Bene M, Mattei L, et al. Fusion imaging for intra-operative ultrasound-based navigation in neurosurgery. J Ultrasound. 2014;17(3):243-251.

28. Prada F, Del Bene M, Mattei L, et al. Preoperative magnetic resonance and intraoperative ultrasound fusion imaging for real-time neuronavigation in brain tumor surgery. Ultraschall Med. 2015;36(2):174-186.

29. Prada F, Mattei L, Del Bene M, et al. Intraoperative cerebral glioma characterization with contrast enhanced ultrasound. BioMed Res Int. 2014;2014. (doi:10.1155/2014/484261) 
30. Prada F, Perin A, Martegani A, et al. Intraoperative contrast-enhanced ultrasound for brain tumor surgery. Neurosurgery. 2014;74(5):542-552.

31. Unsgaard G, Ommedal S, Muller T, Gronningsaeter A, Nagelhus Hernes TA. Neuronavigation by intraoperative three-dimensional ultrasound: initial experience during brain tumor resection. Neurosurgery. 2002;50(4):804-812; discussion 812

32. Serra C, Stauffer A, Actor B, et al. Intraoperative high frequency ultrasound in intracerebral high-grade tumors. Ultraschall Med. 2012;33(7):E306-E312.

33. Prada F, Del Bene M, Casali C, et al. Intraoperative navigated angiosonography for skull base tumor surgery. World Neurosurg. 2015;84(6):1699-1707.

34. Mauri G, Cova L, De Beni S, et al. Real-time US-CT/MRI image fusion for guidance of thermal ablation of liver tumors undetectable with US: Results in 295 cases. Cardiovasc Intervent Radiol. 2015;38(1):143-151.

35. Renovanz M, Hickmann AK, Henkel C, Nadji-Ohl M, Hopf NJ. Navigated versus non-navigated intraoperative ultrasound: is there any impact on the extent of resection of high-grade gliomas? A retrospective clinical analysis. J Neurol Surg A Cent Eur Neurosurg. 2014;75(3):224-230.

36. Le Roux PD, Berger MS, Wang K, Mack LA, Ojemann GA. Low grade gliomas: comparison of intraoperative ultrasound characteristics with preoperative imaging studies. J Neuro-Oncol. 1992;13(2):189-198.

37. Unsgård G, Solheim $O$, Lindseth F, Selbekk T. Intra-operative imaging with 3D ultrasound in neurosurgery. Acta Neurochir (Wien). 2011:181-186. (doi:10.1007/978-3-211-99651-5_28).

38. Unsgaard G, Selbekk T, Brostrup Müller T, et al. Ability of navigated 3D ultrasound to delineate gliomas and metastases-comparison of image interpretations with histopathology. Acta Neurochir (Wien). 2005;147(12):1259-1269.

39. Jung TY, Jung S, Kim IY, et al. Application of neuronavigation system to brain tumor surgery with clinical experience of 420 cases. Minim Invasive Neurosurg. 2006;49(4):210-215.

40. Gerganov VM, Samii A, Akbarian A, Stieglitz L, Samii M, Fahlbusch R. Reliability of intraoperative high-resolution $2 \mathrm{D}$ ultrasound as an alternative to high-field strength MR imaging for tumor resection control: a prospective comparative study. J Neurosurg. 2009;111(3):512-519.

41. Ulrich NH, Burkhardt JK, Serra C, Bernays RL, Bozinov O. Resection of pediatric intracerebral tumors with the aid of intraoperative real-time 3-D ultrasound. Childs Nerv Syst. 2012;28(1):101-109.
42. Solheim O, Selbekk T, Jakola AS, Unsgård G. Ultrasound-guided operations in unselected high-grade gliomas-overall results, impact of image quality and patient selection. Acta Neurochir. 2010;152(11):1873-1886.

43. Moiyadi AV, Shetty PM, Mahajan A, Udare A, Sridhar E. Usefulness of three-dimensional navigable intraoperative ultrasound in resection of brain tumors with a special emphasis on malignant gliomas. Acta Neurochir. 2013;155(12):22172225.

44. Šteňo A, Hollý V, Mendel P, et al. Navigated 3D-ultrasound versus conventional neuronavigation during awake resections of eloquent low-grade gliomas: a comparative study at a single institution. Acta Neurochir (Wien). 2017:1-12. doi: http://dx.doi.org/10.1007/s00701-017-3377-8.

45. Coburger J, Scheuerle A, Kapapa T, et al. Sensitivity and specificity of linear array intraoperative ultrasound in glioblastoma surgery: a comparative study with high field intraoperative MRI and conventional sector array ultrasound. Neurosurg Rev. 2015;38(3):499-509.

46. Chang SM, Parney IF, Mcdermott M, et al. Perioperative complications and neurological outcomes of first and second craniotomies among patients enrolled in the Glioma Outcome Project. J Neurosurg. 2003;98(6):11751181.

47. Cordella R, Nava S, Prada F, Agnoletti A, Legnani F, Dimeco F. Ultrasound guided mini-invasive tailored approach and intraoperative neurophysiological monitoring: a synergistic strategy for the removal of tumors near the motor cortex. A preliminary experience. J Neurosurg Sci. 2018. (doi:10.23736/S03905616.16.03565-7).

48. Fecci PE, Babu R, Adamson DC. Editorial: Turning fluorescence into black and white. J Neuroisurg. 2015;122(6):1356-1359.

49. Prada F, Del Bene M, Rampini A, et al. Intraoperative strain elastosonography in brain tumor surgery. Oper Neurosurg. 2018. (doi: 10.1093/ons/ opy323).

50. Del Bene M, Perin A, Casali C, et al. Advanced ultrasound imaging in glioma surgery: beyond gray-scale B-mode. Front Oncol. 2018;3;8:576. (doi 10.3389/fonc.2018.00576).

51. Prada F, Vitale V, Del Bene M, et al. Contrast-enhanced MR Imaging versus Contrast-enhanced US: A Comparison in Glioblastoma Surgery by Using Intraoperative Fusion Imaging. Radiology. 2017;285(1):242-249. 